\title{
Nanogel-based delivery of mycophenolic acid ameliorates systemic lupus erythematosus in mice
}

\author{
Michael Look, ${ }^{1}$ Eric Stern, ${ }^{1}$ Qin A. Wang, ${ }^{1}$ Leah D. DiPlacido, ${ }^{2}$ Michael Kashgarian, ${ }^{3}$ \\ Joe Craft, ${ }^{2,4}$ and Tarek M. Fahmy',5 \\ 1Department of Biomedical Engineering, ${ }^{2}$ Department of Immunobiology, ${ }^{3}$ Department of Pathology, ${ }^{4}$ Department of Internal Medicine - \\ Section of Rheumatology, and 5Department of Chemical and Environmental Engineering, Yale University, New Haven, Connecticut, USA.
}

\begin{abstract}
The ability to selectively inactivate immune cells with immunosuppressants is a much sought-after modality for the treatment of systemic lupus erythematosus and autoimmunity in general. Here, we designed and tested a novel nanogel drug delivery vehicle for the immunosuppressant mycophenolic acid (MPA). Treatment with MPA-loaded nanogels increased the median survival time (MST) of lupus-prone NZB/W F1 mice by 3 months with prophylactic use (MST was 50 weeks versus 38 weeks without treatment), and by 2 months when administered after the development of severe renal damage (MST after proteinuria onset was 12.5 weeks versus 4 weeks without treatment). Equivalent and greater doses of MPA administered in buffer were not efficacious. Nanogels had enhanced biodistribution to organs and association with immune cells. CD4-targeted nanogels yielded similar therapeutic results compared with nontargeted formulations, with protection from glomerulonephritis and decreases in IFN- $\gamma$-positive CD4 T cells. DCs that internalized nanogels helped mediate immunosuppression, as they had reduced production of inflammatory cytokines such as IFN- $\gamma$ and IL-12. Our results demonstrate efficacy of nanogel-based lupus therapy and implicate a mechanism by which immunosuppression is enhanced, in part, by the targeting of antigen-presenting cells.
\end{abstract}

\section{Introduction}

In systemic lupus erythematosus (SLE), multiorgan injury results from the dysregulated activation of adaptive and innate immune cell subsets, which leads to autoantibody deposition in tissues and subsequent inflammatory damage (1). Pathology reflects a fundamental loss of self-tolerance where CD4 T and B lymphocytes are critical for autoantibody production (2), and dendritic cells (DCs) produce inflammatory cytokines and help stimulate autoreactive lymphocytes (3-5). Current treatment strategies are primarily intended to attenuate these cellular responses and thus prevent the spontaneous recurrence of lupus flares and worsening of disease, such as renal failure (6). However, limitations persist. Small-molecule drugs typically require lifelong, daily dosing (7), and some are particularly toxic, such as cyclophosphamide (8) and glucocorticoids (9). Effective treatment outcomes are complicated by patients' nonadherence rates to therapeutic regimens that are as high as $40 \%-50 \%$ (10). Biological therapies that have been tested in recent clinical trials provided marginal or no benefit despite being tailored for cell-specific suppression: CTLA4Ig (11) and rituximab (12) were ineffective for remission induction therapy, whereas belimumab reduced the 1-year incidence of disease flares by only about $10 \%(13)$.

We postulate that nanoparticulate drug delivery systems can improve lupus therapy because they can be designed to effectively target small-molecule drugs or other agents to immune cells that contribute to disease. Such particulate modalities have been actively investigated for cancer therapies (14) and vaccination (15), but their efficacy in achieving therapeutic immunosuppression in lupus has been mostly unexplored. Published reports

Conflict of interest: The authors have declared that no conflict of interest exists. Citation for this article: J Clin Invest. 2013;123(4):1741-1749. doi:10.1172/JCI65907. on lupus models are limited to demonstrations of nanoparticles that can travel to sites of tissue inflammation, such as the kidneys (16), where they have potential utility as a contrast agent for diagnostic imaging (17). But little is known about how nanoparticles interact with immune cell subsets in lupus, or if these interactions could be therapeutically exploited.

In this work, we developed a nanoparticle that consists of a biodegradable, gel-like core containing cyclodextrins that are enclosed by a lipid bilayer (18). This unique formulation is a nanogel (19) that resembles a hybrid of two clinically used platforms, liposomes (20) and biodegradable polymer matrices (21), and adopts favorable biophysical features of each. Its liposomal-like properties are amenable for facile encapsulation of a broad range of hydrophilic and hydrophobic molecules, while its polymeric core helps enhance or retain drug loading and imparts stability. Mycophenolic acid (MPA), whose prodrug form mycophenolate mofetil (MMF) is used in lupus patients $(22,23)$, was encapsulated inside nanogels. Although MPA is a hydrophobic drug with a reported partition coefficient (log $P$ value) of 3.88 (24), the use of cyclodextrins in the particle formulation permits its robust aqueous solubilization and encapsulation.

MPA-loaded nanogels effectively treated murine lupus, extending the mean survival time by 3 months when used prophylactically and by 2 months when given after the onset of elevated proteinuria. Strikingly, equivalent doses of MPA administered in saline (not particulate-bound, and referred to as "free MPA") provided no benefit. We also evaluated the efficacy of targeting $\mathrm{T}$ cells using particles functionalized with CD4 antibodies, and found no additional increases in mean survival times compared with particles without CD4 (referred to as "nontargeted"). Investigations on how various immune cells responded to therapy revealed that DCs had attenuated production of inflammatory cytokines and stimu- 
A

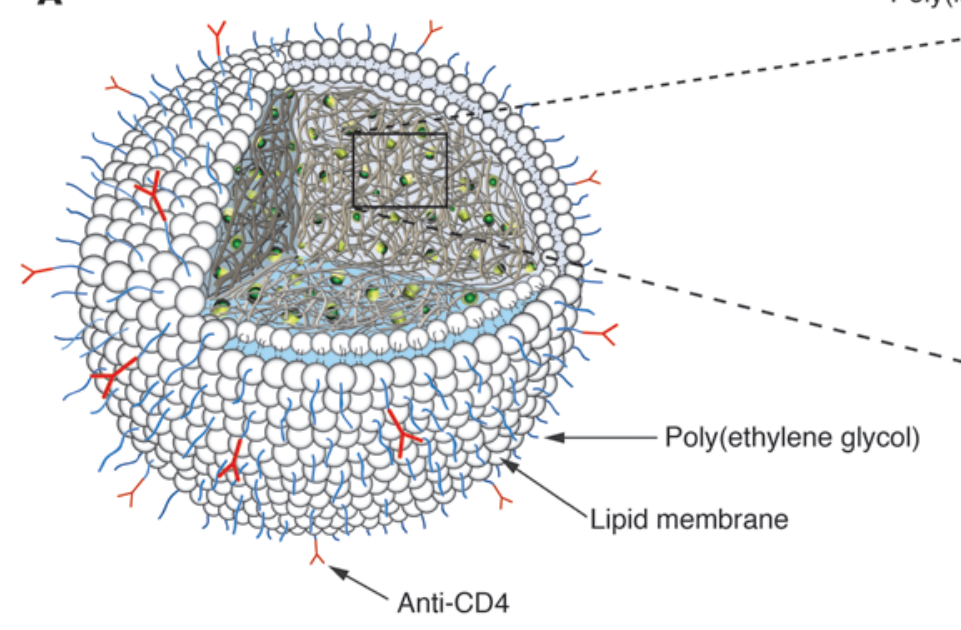

Poly(lactic acid-co-ethylene glycol-co-lactic acid)

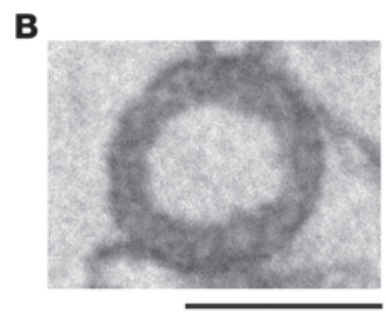

B
C

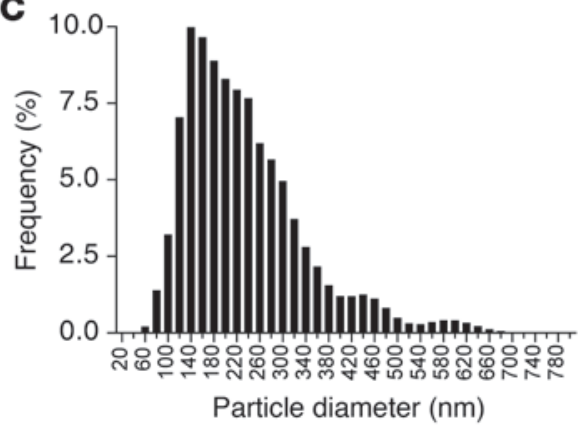

D

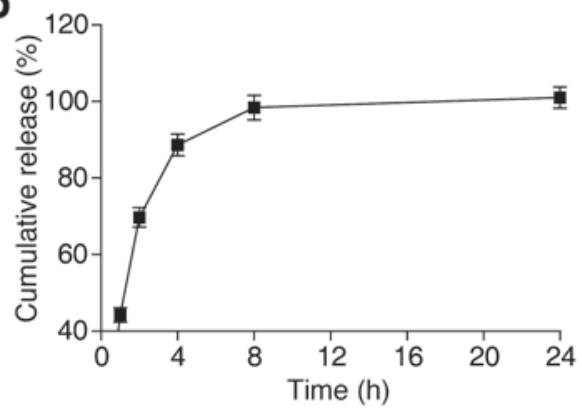

\section{Figure 1}

Fabrication and characterization of MPA-loaded nanogels. (A) Nanogels were fabricated by remotely loading liposomes with MPA solubilized within cyclodextrin, oligomers of lactic acid-poly(ethylene glycol) that were terminated with an acrylate end group, and Irgacure 2959 photoinitiator. Particles were then exposed to ultraviolet light to induce photopolymerization of the PEG oligomers. (B) Transmission electron microscopic image of nanogels. Nanogels were fixed in Epon resin, sectioned at $60 \mathrm{~nm}$, and then stained with osmium tetroxide. Scale bar: $200 \mathrm{~nm}$. (C) Hydrodynamic size distribution. The nanogel size was measured in PBS ( $\mathrm{pH}$ 7.4) using single-particle motion-tracking methods. The median diameter was approximately $203 \mathrm{~nm}$. (D) MPA release profile from nanogels. The cumulative release of drug was performed by dialyzing particles against PBS at $37^{\circ} \mathrm{C}$. The mean amount of release is shown, with error bars indicating the standard deviation of triplicate measurements from 1 representative experiment that was repeated at least 3 times. The average loading of MPA in particles was approximately $6.65 \pm 3.82 \mu \mathrm{g}$ $\mathrm{MPA} / \mathrm{mg}$ particle (mean \pm standard deviation) among all batches used in experiments.

latory surface markers in vitro. Modulation of DCs was suggestive of the therapeutic mode of action, for although lymphodepletion was not observed, 2 -fold decreases in the percentage of splenic IFN- $\gamma$-producing CD4 $\mathrm{T}$ cells occurred in vivo. This ability to suppress immune cell activity demonstrates the therapeutic power of using nanogels to treat murine lupus.

\section{Results}

Synthesis and in vitro characterization of a nanogel-based particle. Nanogels were fabricated by remotely loading liposomes with a diacrylate-terminated coblock polymer of poly(lactic acid-co-ethylene glycol) (25), Irgacure 2959 photoinitiator, and MPA complexed within nonmethylated $\beta$-cyclodextrin (Figure 1A and ref. 18). Subsequent photopolymerization under UV light promoted cross-linking between the acrylated macromers and gelation of the particle interior into a stable matrix (Supplemental Figure 1; supplemental material available online with this article; doi:10.1172/ JCI65907DS1). Transmission electron microscopic images revealed that lipid-specific osmium tetroxide staining of 60 -nm sectioned nanogels had a localized staining pattern confined to their exterior membrane (Figure 1B). The average hydrodynamic diameter was $225 \mathrm{~nm}$, with a median of $203 \mathrm{~nm}$ and a mode of $141 \mathrm{~nm}$ (Figure 1C). The average MPA loading (among all lots of particles used in this study) was approximately $6.65 \pm 3.82 \mu \mathrm{g} \mathrm{MPA} / \mathrm{mg}$ particle (mean \pm standard deviation), with an encapsulation efficiency of approximately $3.79 \%$. Drug was released over several hours (Figure 1D) and retained its potency (Supplemental Figure 2).

We next explored whether nanogels could be targeted against CD4 T cells to suppress their proliferation. In lupus, CD4 T cells stimulate autoreactive B cells $(26,27)$ and cause cytolytic damage in inflamed tissue (28). Depletion of T cells ameliorates disease in murine models (29-33), and we incorporated this strategy by covalently attaching a CD4 antibody (clone RM4-4) to nanogels. Cell binding was verified in vitro (Supplemental Figure 3), whereby purified CD4 $\mathrm{T}$ cells were incubated with particles for 20 minutes, washed to remove everything unbound to cells, and then cultured 
A
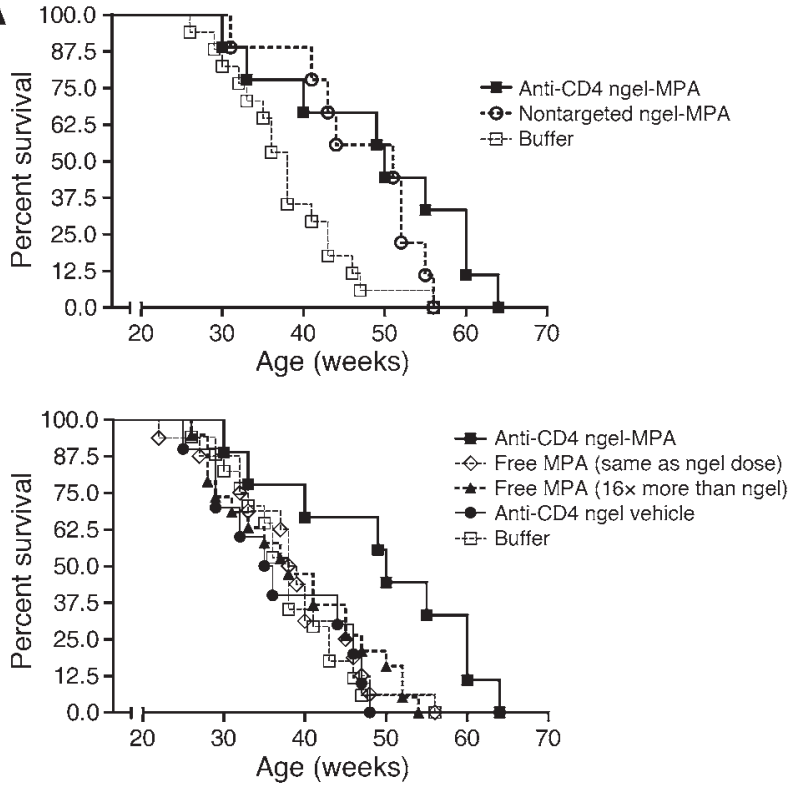

B

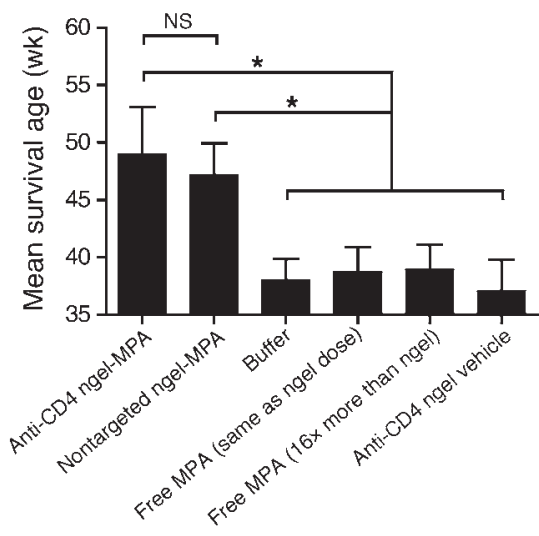

for 4 days with CD3 and CD28 antibodies. Only targeted, MPAloaded nanoparticles inhibited proliferation; in contrast, nontargeted nanoparticles and free MPA failed to do so.

MPA-loaded nanogels enhance survival of lupus-prone NZB/W F1 mice. The in vivo efficacy of nanogels was tested in female New Zealand black/New Zealand white (NZB/W F1) mice, which develop autoimmunity that resembles human SLE and is characterized by antinuclear autoantibodies (ANA), abnormal lymphoproliferation, and fatal glomerular nephritis. We first administered particles prophylactically, where weekly dosing was started at 18 to 20 weeks of age, before the onset of proteinuria. The MPA dosage was $0.625 \mathrm{mg}$ of MPA per kilogram of animal body weight (mpk). Control mice that received only saline buffer had a median survival age of 38 weeks, but treatment with CD4-targeting particles extended survival to 50 weeks $(P<0.0083)$, and to 51 weeks with nontargeted particles $(P<0.0304)$ (Figure 2). MPA delivery in nanogels was critical for achieving therapeutic benefit, with nontargeted particles being as effective as CD4-targeted ones. Equivalent dosing of free MPA at $0.625 \mathrm{mpk}$ did not extend survival, nor did 16-fold more of free drug at $10 \mathrm{mpk}$. These results demonstrate the therapeutic advantage of using nanoparticles, as published reports have shown that much larger and more frequent free MPA regimens of 30 to 100 mpk every day are used for treating murine lupus $(34,35)$.

\section{Figure 2}

Nanogels (ngel) extend lupus survival better than equivalent and 16-fold greater doses of free MPA. NZB/W F1 mice were treated with a lifelong weekly dose of MPA, at $0.625 \mathrm{mpk}$, using nanogels beginning at 18 to 20 weeks of age. MPA that was not encapsulated within particles, which is referred to as "free MPA," was dosed at $0.625 \mathrm{mpk}$ or at a 16 times greater dose at $10 \mathrm{mpk}$; vehicle controls are particles without MPA encapsulated, dosed at approximately $5 \mathrm{mg}$ of particle per animal. The (A) Kaplan-Meier survival curve and (B) mean survival age are shown. Statistical analysis was performed using the log-rank test, with significance comparisons among groups indicated in $\mathbf{B} ;{ }^{*} P<0.05$, with error bars in $\mathbf{B}$ representing the standard error measurement. The sample size is 9-19 animals per group. The use of CD4-targeted and nontargeted nanogels extended the mean survival time by approximately 2 to 3 months, whereas free drug doses were not efficacious. CD4-targeted and nontargeted treatment groups were not significantly (NS) different when compared with each other.

Particles given after the onset of proteinuria were also effective; survival time lasted 12 weeks with particles compared with only 4 weeks with buffer $(P<0.0198)$ (Supplemental Figure 4).

Nanogels protected against nephritis. Mice receiving prophylactic treatment had delayed onset of proteinuria (Figure 3A) and presence of leukocyte esterases in the urine (Figure 3B) compared with free drug and buffer treatments. Moreover, the percentage of mice with abnormally elevated blood urea nitrogen (BUN) levels (greater than $18-29 \mathrm{mg} / \mathrm{dl}$ ), which is an indicator of impaired renal function, was lower in 36- to 40-week-old mice (Figure 3C). Consistent with these observations, H\&E-stained sections of kidneys from particle-treated groups had reduced glomerular damage (Figure 3D and Supplemental Figure 5).

We also evaluated the acute hematological and organ toxicity of nanogels in C57BL/ 6 mice. After 4 daily doses of $0.625 \mathrm{mpk}$ MPA in particles were administered, complete blood counts (CBCs) and clinical chemistries for liver and kidney function were measured 4,7 , and 14 days after the first dose. White blood cell, platelet, hemoglobin, and hematocrit levels were within normal physiological ranges (Supplemental Figure 6, A-D). Furthermore, no liver or renal toxicities were observed. Body weight and serum concentrations for alkaline phosphatase (ALKP), alanine transferase (ALT), total bilirubin (tBIL), and BUN were normal (Supplemental Figure 7, A-E).

Nanogels associate with immune cells in vivo. We originally hypothesized that CD4 targeting would augment the ability of particles to abolish T-dependent autoimmunity. However, CD4-targeting and nontargeting particles provided similar efficacy in vivo (Figures 2 and 3). To explain why, we compared their biodistribution using rhodamine, a fluorescent label. Regardless of targeting, nanogels had greater accumulation in the spleen, kidneys, liver, heart, lung, and pancreas compared with free rhodamine (Figure 4A). This trend is consistent with other reports which show that particulate drug delivery systems can increase the bioavailability of compounds (21). In the spleen, particles localized to the red pulp (Figure 4B) and had some infiltration into the $\mathrm{T}$ cell zone (Figure 4C) after 2 to 3 hours. T and B220+ cells associated with particles, with the greatest binding (based on the percentage of the cell subset) observed with DCs, macrophages, and plasmablasts (Figure 4D). Additional particle accumulations were observed with cells in the bone marrow (Supplemental Figure 8).

Particulate therapy partially modulates T cell phenotype without causing lymphocytopenia. White blood cell counts were not reduced 
A
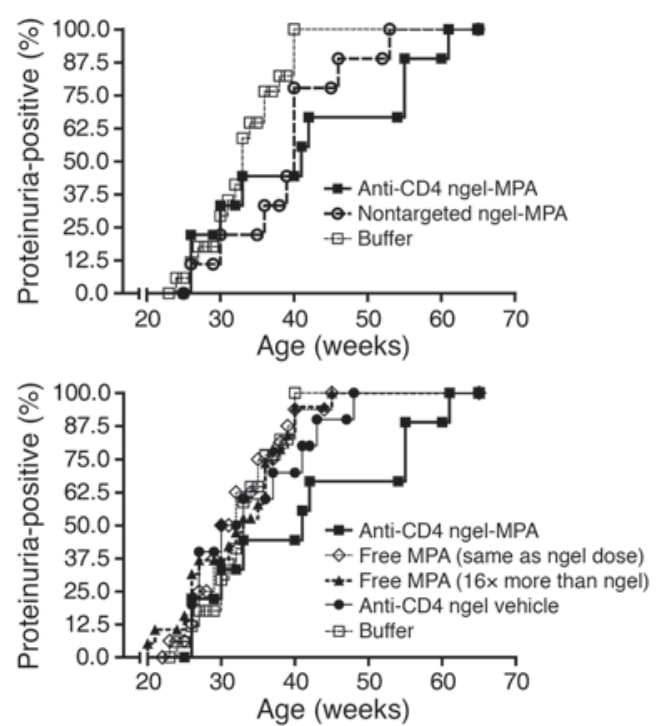

B
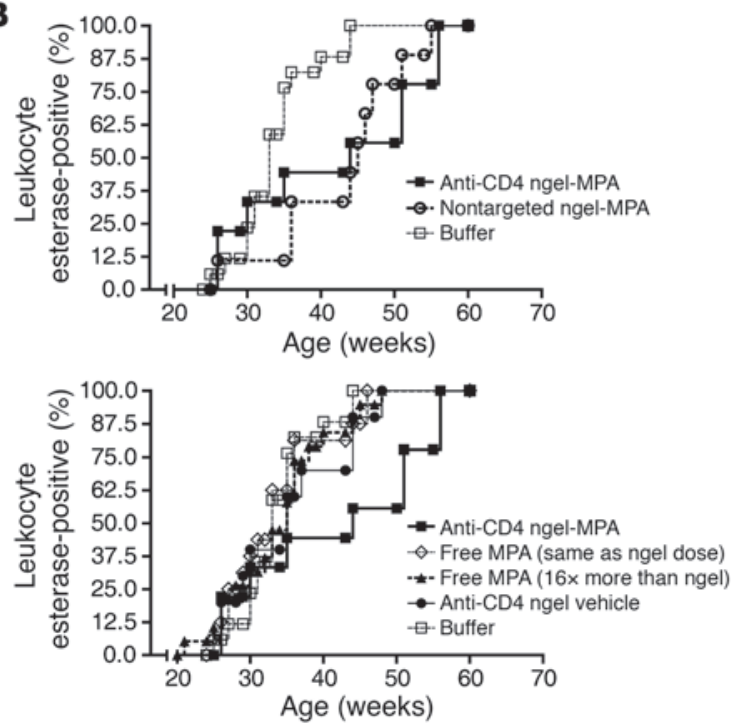

C

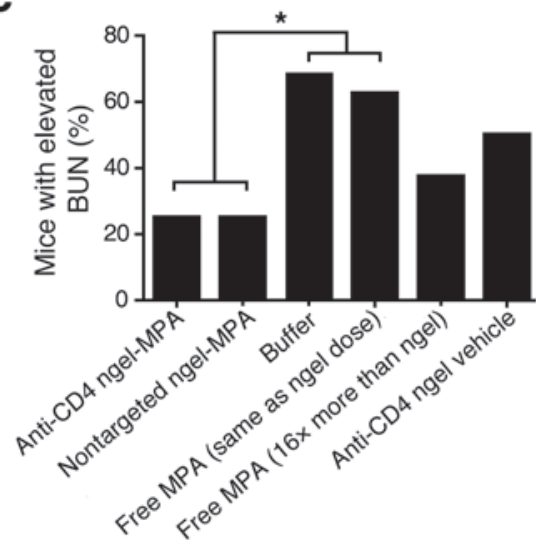

D

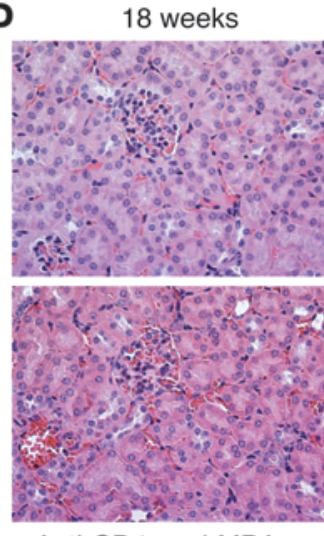

Anti-CD4 ngel-MPA

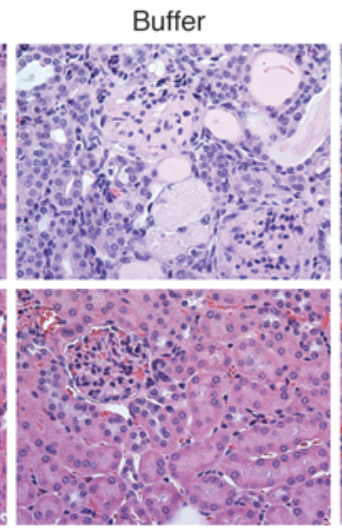

Nontargeted ngel-MPA

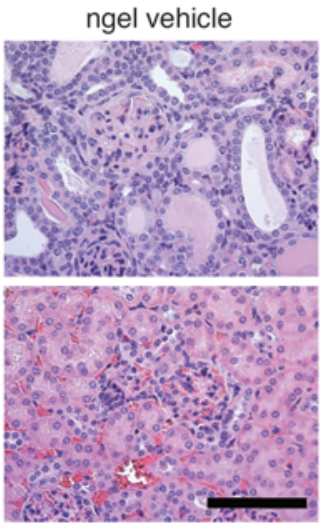

Free MPA

16x more than ngel)

\section{Figure 3}

Nanogel therapy delays the onset of renal damage. NZB/W F1 mice were monitored for the onset and severity of renal damage; weekly treatments with nanogels beginning at 18 to 20 weeks of age resulted in the delayed onset of (A) proteinuria (defined as $\geq 300 \mathrm{mg} / \mathrm{dl}$ of protein in urine), and (B) the presence of leukocyte esterase content in the urine (which corresponds to $>10$ leukocytes/ $\mu$ in the urine), as determined by dry reagent Uristix assay. For urinalysis (A and $\mathbf{B}$ ), treatment with CD4-targeted or nontargeted MPA particles compared with free MPA, buffer, and vehicle groups was statistically significant $(P<0.05$ by 1 -way ANOVA with Bonferroni's multiple comparison test), though no significant difference was detected between CD4-targeted MPA particles compared with nontargeted ones. The sample size is 9-19 animals per group. (C) Renal function was assessed by BUN in mice at 36 to 40 weeks of age; an elevated BUN was defined as greater than the physiological reference range of 18 to $29 \mathrm{mg} / \mathrm{dl}$. The sample size is 8 mice per group. ${ }^{*} P<0.05$ by 2-tailed Student's $t$-test. (D) Representative images of H\&E-stained sections of kidneys from mice at 36 to 40 weeks of age. Mice that received nanogel MPA therapy had reduced nephritis. Scale bar: $500 \mu \mathrm{m}$.

after 4 consecutive daily doses of nanogels in wild-type mice (Supplemental Figure 6A). This observation led us to suspect that particulate-based immunosuppression occurs by modulating immune cell function or activation state rather than by lymphodepletion. Although abolishing autoreactive germinal center responses is one method to ameliorate lupus (26), this was not completely accomplished with particulate therapy. In 36- to 40-week-old NZB/W F1 mice, anti-dsDNA antibody serum titers were only marginally reduced, and not eliminated (Supplemental Figure 9). No differences were observed in the percentage of splenic germinal center B cells or CD4 T follicular helper cells (Supplemental Figure 10), though there were mild reductions in the percentages of peripheral blood CD $138^{\text {hi }} \mathrm{B} 220^{\text {lo }}$ antibodysecreting B cells (Supplemental Figure 11A).

Despite the persistence of autoantibodies, inflammatory and activated (CD44hi) $\mathrm{T}$ cells were diminished. Mild reductions in the percentage of activated peripheral blood and splenic CD4 $\mathrm{T}$ cells $\left(\mathrm{CD} 62 \mathrm{~L}^{\text {lo }} \mathrm{CD} 44^{\mathrm{hi}}\right.$ ) were observed (Supplemental Figure 11B and Supplemental Figure 12, A and B). The percentage of splenic IFN- $\gamma$-producing CD $4 \mathrm{~T}$ cells was significantly reduced by approximately 2-fold (Supplemental Figure 12C), from 10\% of CD4 $\mathrm{T}$ cells in untreated controls to $5 \%$ with particle treatment. However, there were no effects on the percentage of CD4 $\mathrm{T}$ regulatory (Treg) cells (Supplemental Figure 12D). 
A
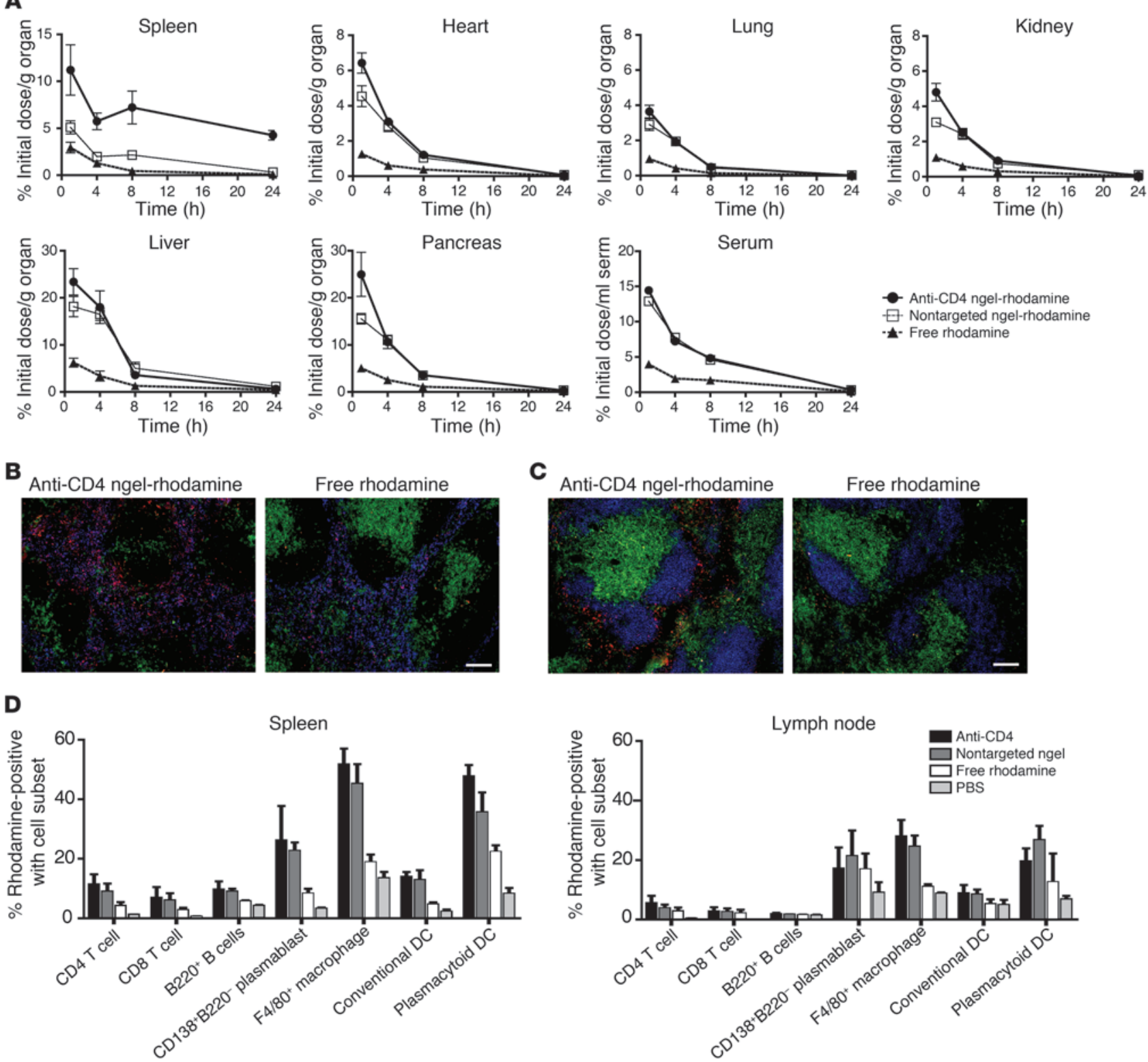

Figure 4

Biodistribution of nanogels. Rhodamine-loaded nanogels were injected intraperitoneally into 12-week-old NZB/W F1 mice. (A) Organ-specific fluorescence was measured and rhodamine encapsulated in nanoparticles (both CD4-targeted and nontargeted) had statistically significantly higher accumulations than free rhodamine. Targeted particles had statistically significantly higher accumulation than nontargeted particles as well ( $P<0.05$ by Student's 2-tailed $t$ test for all organs). Trace amounts of fluorescence could be detected in particle groups at 48 and 72 hours (not shown). Error bars represent the standard deviation. The sample size is 3 or 4 mice per group. The localization of rhodamine nanogels within the spleen was also analyzed by immunofluorescence histology (B and $\mathbf{C}$ ). Histological analysis of spleens was performed 1 hour after rhodamine nanoparticle (red) injection, and stained for, in B, CD4 T cells (green) and F4/80 macrophages (blue); or in C, CD4 T cells (green), and CD19 B cells (blue). Scale bars (B and C): $100 \mu \mathrm{m}$. (D) Rhodamine-loaded nanogels were injected intraperitoneally into C57BL/6 mice that had been vaccinated with sheep red blood cells 7 days earlier. Approximately $2-3$ hours later, the percentage of rhodamine-positive immune cells in the spleen and the lymph nodes (pooled inguinal, cervical, and brachial) was quantified by flow cytometry. CD4-targeted and nontargeted particles had greater binding with immune cells, particularly in the spleen compared with lymph nodes. $P<0.05$ by 1 -way ANOVA comparison between particles and free rhodamine for both spleen and lymph node. Error bars represent the standard error measurement, with a sample size of 4 mice per group.

Nanogels suppress inflammatory responses in DCs. The in vivo effects on $\mathrm{T}$ cells led us to consider whether DCs had impaired stimulatory capacity, since lymphodepletion was not identified in CBCs (Supplemental Figure 6A) to be a dominant mechanism for immu- nosuppression. The involvement of DCs is implicated by their internalization of nanogels (Figure 4D, Figure 5A, and Supplemental Figure 8$)$ and in reports showing that free $(36,37)$ and particulate-based MPA (38) suppresses DC inflammatory responses. 
A

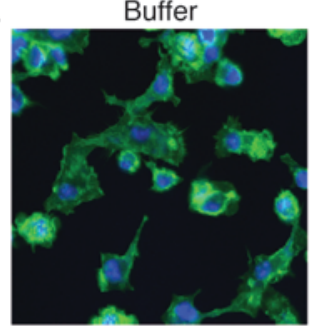

+Nanoparticles

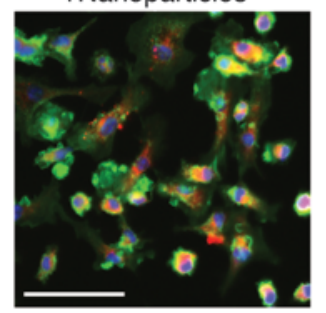

B
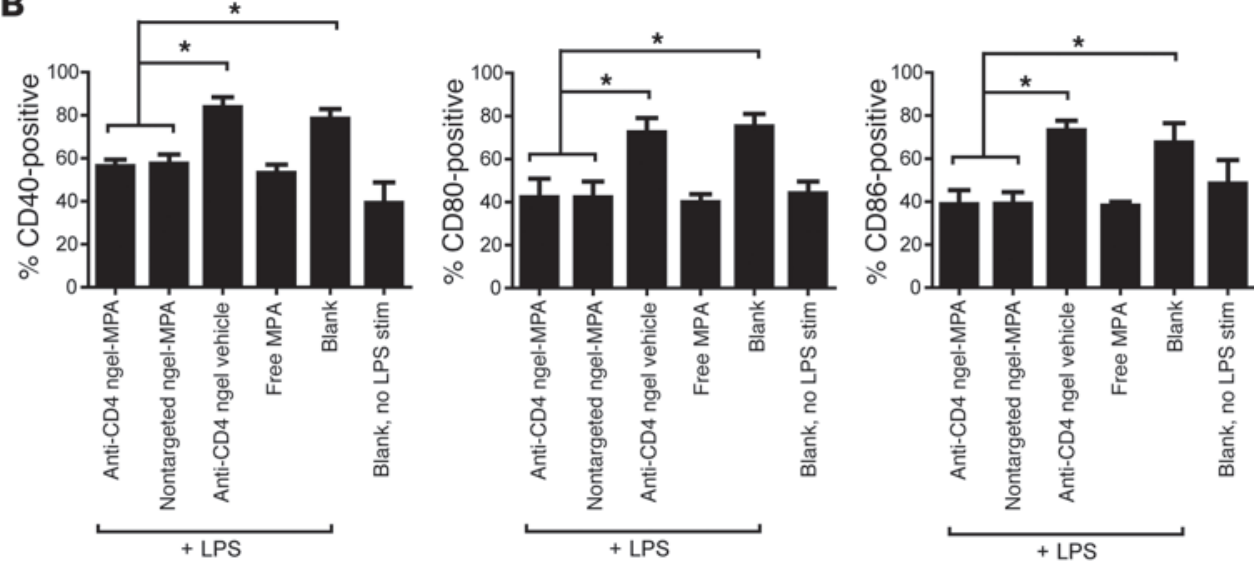

D

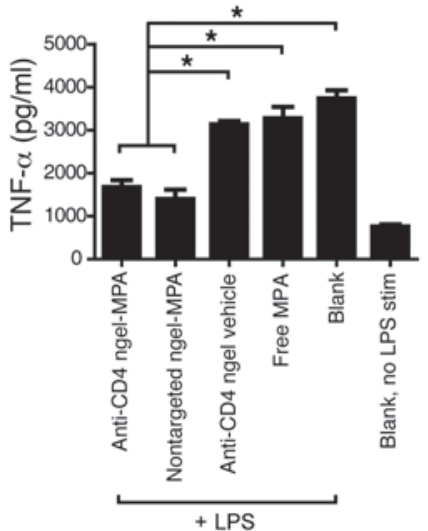

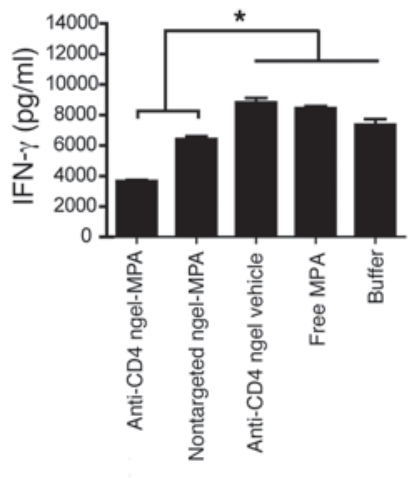

Figure 5

DCs internalize nanogels and have suppressed inflammatory phenotype and allostimulatory capability. (A) BMDCs were cultured in vitro for 7 days and then incubated with rhodamine-labeled nanogel particles for 1 hour. Confocal images were acquired from BMDCs treated with nanoparticles (red) and stained with actin-specific phalloidin-Alexa 488 (green) and DNA-specific TO-PRO-3 (blue). Scale bar: $50 \mu \mathrm{m}$. (B and C) BMDCs were treated with $125 \mathrm{ng} / \mathrm{ml}$ of MPA beginning on day 1 of culture, and then on day 6 , BMDCs were challenged with $50 \mathrm{ng} / \mathrm{ml}$ of LPS for 18 hours. The (B) BMDC surface marker expression and (C) cytokine secretion were decreased in cells treated with MPA. Results are the average of 3 separate experiments, with error bars representing the standard error measurement. (D) CD11 $\mathrm{c}^{+} \mathrm{DCs}$ from treated mice have reduced allostimulatory capability. Female BALB/c mice were injected interperitoneally with $0.125 \mathrm{mpk}$ of MPA on days 0,3 , and 7 . On day 8 , CD11c ${ }^{+}$cells from the spleen were isolated, irradiated with $30 \mathrm{~Gy}$, and then cocultured for 4 days with CD4 T cells from female C57BL/6 mice, at a ratio of $1 \times 10^{5} \mathrm{DCs}$ to $5 \times 10^{5} \mathrm{~T}$ cells. IFN- $\gamma$ production was lower using DCs from mice that received MPA-loaded nanogels. Error bars represent the standard deviation, with triplicate measurements from 1 representative experiment shown. This experiment was performed twice, with similar trends. ${ }^{*} P<0.05$ by ANOVA comparison with Bonferroni's post test.

In agreement with these observations, splenic conventional DCs from nanogel-treated mice had slight reductions in their expression of CD40 and MHC class II (Supplemental Figure 13), though this trend was not seen in plasmacytoid DCs or macrophages.

The suppression of DC activation markers by MPA is reversible in vitro and requires the continued presence of MPA in media to maintain attenuated responses (36). Hence, the slight reduction in CD40 and MHC class II in vivo may be a consequence of the intermittence of weekly dosing. Much greater suppression was observed with constant nanogel exposure to cells in vitro. Bone marrow-derived DCs (BMDCs) treated with $125 \mathrm{ng} / \mathrm{ml}$ of MPA in nanogels had substantially decreased surface expression of CD40, CD80, and CD86 (Figure 5B), as well as MHC class I and MHC class II (Supplemental Figure 14), and also had decreased production of the inflammatory cytokines IFN- $\gamma$, IL-12, and TNF- $\alpha$ (Figure $5 \mathrm{C}$ ). Allogeneic CD4 T cells that were cocultured with these DCs were less proliferative (Supplemental Figure 15A) and had some increased differentiation into FOXP3 ${ }^{+} \mathrm{CD} 25^{+}$Treg cells (Supplemental Figure 15B). Furthermore, CD $11 \mathrm{c}^{+} \mathrm{DCs}$ isolated from wild-type mice that were treated with nanogels elicited weaker production of IFN- $\gamma$ from allogeneic CD4 T cells (Figure 5D).

We also explored whether nanogels affected IFN- $\alpha$ production by plasmacytoid DCs in vitro. Plasmacytoid DCs that were incubated with nanogels for 30 minutes and then challenged with $\mathrm{CpG}$ oligonucleotides had reduced production of IFN- $\alpha$ (Supplemental Figure 16). Our results implicate MPA delivery within nanogels to 
DCs as a method to attenuate aberrant inflammatory responses that contribute to lupus autoimmunity.

\section{Discussion}

Clinically available therapies for lupus suffer from refractoriness in some cases $(23,39)$, typical requirements for daily dosing $(7)$, high toxicity $(8,9)$, or high nonadherence rates among patients (10). We reasoned that localized drug delivery to immune cells could greatly mitigate these challenges, and thus used a nanogel formulation to achieve better therapeutic immunosuppression. Weekly nanogel treatments prolonged lupus survival, whereas equivalent, and at least an order of magnitude greater, dose of free MPA did not. Toxicological analyses with CBCs and clinical chemistry tests demonstrated the safety of this nanoscale platform.

The suppression of immune cells is the principal mechanism of action of many current and investigative drugs for lupus. Therefore, nanogels are attractive because of their intrinsic abilities to enable greater systemic accumulations of their cargo and to bind more immune cells in vivo than free fluorescent tracer, which, we reason, permits high, localized concentrations of MPA. In the spleen, particles associated with approximately $10 \%$ of T and B220 B cells, $15 \%$ of conventional DCs, and $40 \%$ of plasmacytoid DCs. Although CD4 targeting moderately increased the systemic accumulation of particles, it conferred no additional survival benefit compared with nontargeted particles. The lack of benefit with CD4 targeting could be explained by the fact that DCs avidly internalized particles and had suppressed inflammatory responses, thereby disrupting lymphocyte stimulation or tissue damage. This view is supported by studies on the genetic deletion of CD11c DCs in MRLFaslpr mice, which results in diminished disease but is insufficient to prevent the initiation and expansion of $\mathrm{T}$ and $\mathrm{B}$ cell autoreactivity (3). At the dosage of nanogels tested, such a DCcentric mechanism of therapy is consistent with our finding that germinal center responses are not abolished in 36- to 40-week-old mice receiving prophylactic therapy. Survival benefits are accomplished even when nanogel treatment is started after the onset of proteinuria, which occurs after the expansion of autoreactive lymphocytes and elevation of autoantibody titers in NZB/W F1 mice. Nonetheless, the contribution of CD4 T cells in lupus is well documented (2, 29, 40-42), and further optimization of nanoparticles so that they better or more selectively target $T$ cells remains important. $\mathrm{T}$ cell depletion, in conjunction with the modulation of DCs so that they are less potent stimulators or become tolerogenic, could increase the duration of remission by suppressing $\mathrm{T}$ cell-mediated autoimmunity through two distinct but additive pathways. Thus, the balance of nanoparticle targeting to both cell types is crucial. Further improvements in formulation or dosing regimen that increase nanoparticle binding to cells could yield even greater efficacy. For example, nanoparticles designed with enhanced penetration to the white pulp of the secondary lymphoid organs may better inhibit $\mathrm{T}$ and $\mathrm{B}$ lymphocyte proliferation.

While we have focused our analysis of nanogel effects on immune cell modulation, it is possible that particles may also impact other cells that are critical to disease. In particular, mesangial cells in the kidney promote the glomerular damage associated with lupus mortality (43), and methods that inhibit these cells represent an alternative, and potentially synergistic, mechanism of therapy. Mesangial cell proliferation is inhibited by MPA (44), and some published studies have demonstrated the treatment of IgA nephropathy in rats using MPA-loaded liposomes (45). Our nanogels have enhanced accumulation in the kidneys, and future investigations warrant a closer study of particle-cell interactions within the renal architecture. Indeed, many nanoparticle systems, including our nanogels, can be flexibly modified for targeted drug delivery to the kidney $(16,17)$, but this therapeutic aspect for lupus has yet to be more fully explored.

This work is a comprehensive study to demonstrate the in vivo efficacy of using nanoparticles containing a clinically relevant drug to treat murine lupus. We show that these survival benefits are uniquely provided by particles, due in part to their internalization by DCs and their subsequent suppression of inflammatory responses. The ability to more potently attenuate lupus autoimmunity with nanogels than is possible with free drug is an advancement for disease treatment strategies, with the potential for broader application to other autoimmune conditions.

\section{Methods}

Fabrication of nanogels. Nanogels were fabricated by remotely loading liposomes with MPA (Sigma-Aldrich) and cross-linkable poly(ethylene glycol) oligomers. To prepare liposomes, a molar ratio mixture of 2:1:0.1 phosphatidylcholine/cholesterol/DSPE-PEG(2000)-amine (Avanti Polar Lipids) in chloroform was evaporated under a nitrogen gas stream and then lyophilized. The resulting dry lipid film was rehydrated with PBS and then extruded 5 times through a 200-nm pore filter (Whatman, GE Healthcare) and then 5 times through a 100-nm pore filter. Liposomes were then surface functionalized with a nondepleting CD4 antibody (clone RM4-4; BD Biosciences) using sulfo-NHS/EDC (Pierce Biotechnology) covalent conjugation, and lyophilized overnight.

Next, $20 \mathrm{mg}$ of MPA in $400 \mu \mathrm{l}$ of methanol was complexed to $100 \mathrm{mg}$ of aminomethacrylate/succinylated $\beta$-cyclodextrin and $300 \mathrm{mg}$ of hydroxypropyl $\beta$-cyclodextrin (CTD Holdings) in PBS under vigorous mixing for 15 minutes at room temperature. Methanol was evaporated from the MPA-cyclodextrin mixture using a nitrogen gas stream. Next, the cyclodextrin-MPA mixture was combined with Irgacure 2959 (Ciba) photoinitiator and a PEG oligomer consisting of 4,000 Da linear poly(ethylene glycol) flanked on each side by approximately 1-2 lactic acid monomers with a terminating acrylate group. The synthesis of this cross-linkable oligomer is described by Sawhney (25)

Lyophilized liposomes were rehydrated with the aqueous MPA-cyclodextrin-Irgacure-PEG mixture. Vigorous mixing was applied for 30 minutes. The liposomes were then cross-linked under a $430 \mathrm{~W}$ UV lamp with UVA light (315-400 nm transmission filter) for 8 minutes on ice to form the nanogel, rinsed with PBS, and pelleted by ultracentrifugation at 98,205 rcf for 1 hour at $4{ }^{\circ} \mathrm{C}$. Nanogels were stored at $-20^{\circ} \mathrm{C}$ until use.

Nanoparticle characterization. The hydrodynamic diameter of nanoparticles in PBS was measured with an NS500 nanoparticle tracking system (NanoSight). For transmission electron microscopy, $60-\mathrm{nm}$ sections of nanogels were stained with osmium tetroxide and then imaged on an FEI Tecnai Biotwin microscope. The amount of MPA loaded in nanoparticles was determined from a known dry weight of particles dissolved in $1 \%$ Triton X-100 in $0.1 \mathrm{M} \mathrm{NaOH}$ for at least 1 hour at $37^{\circ} \mathrm{C}$. MPA concentration was detected by fluorescence, using an excitation wavelength of $340 \mathrm{~nm}$ and an emission wavelength of $450 \mathrm{~nm}$ on a SpectraMax plate reader (Molecular Devices).

Animal studies. For prophylactic treatments, female NZB/W F1 mice (The Jackson Laboratory; stock no. 100008) were given a weekly intraperitoneal injection of nanogels beginning at 18 to 20 weeks of age, with dosing continued until the survival endpoint was reached. Particles were administered by intraperitoneal injection, as opposed to intravenous tail vein injection, to prevent long-term tail vein damage from repeated injections. Mice urine was collected by bladder compression and blood samples were obtained via 
retro-orbital bleeds in isoflurane-anesthetized mice. The survival endpoint was reached when cachexia (more than 5\%-10\% body weight loss) and overall poor condition were visible, at which time the mice were euthanized. For treatments initiated after the development of more severe nephritis, weekly treatments were started in mice that had 2 consecutive daily readings of elevated proteinuria ( $\geq 300 \mathrm{mg} / \mathrm{dl}$ ) based on Uristix (Siemens) measurement.

Flow cytometer analysis. Peripheral blood lymphocytes were collected in $50 \mathrm{U} / \mathrm{ml}$ heparin in PBS and isolated with Ficoll gradient separation. Splenocytes were harvested and red blood cells were removed with ACK lysis buffer. Lymphocytes were blocked with 1\% FBS in PBS, and then stained with the following combinations: T cells (CD4 [clone RM4-5], CD8, CD62L, CD44, PD-1, and CXCR5-biotin); B cells (B220, CD138, IgD, GL-7, PNA-biotin from Vector Laboratories, and MHC II); and innate APCs (F4/80, CD11c, PDCA-1, CD40, CD80, and MHC II; all antibodies from BD Biosciences or eBioscience). Streptavidin Pe-Cy7 (Invitrogen) was used as a detection agent for biotinylated antibodies. Cells were fixed with $2 \%$ paraformaldehyde and then analyzed on an LSRII flow cytometer (BD Biosciences). For in vitro proliferation studies, $\mathrm{T}$ cells were labeled with $0.5 \mu \mathrm{M}$ CFDA-SE (Invitrogen) in PBS for 10 minutes at $37^{\circ} \mathrm{C}$ at a concentration of $4 \times 10^{6}$ cells $/ \mathrm{ml}$, and then washed twice with RPMI-1640 complete media (10 mM HEPES, $1 \mathrm{mM} \mathrm{L}$-glutamine, $100 \mathrm{U} / \mathrm{ml}$ penicillin, $100 \mu \mathrm{g} / \mathrm{ml}$ streptomycin, $50 \mu \mathrm{M} \beta$-mercaptoethanol), with $10 \%$ heat-inactivated FBS. For intracellular IFN- $\gamma$ staining, $1 \times 10^{6}$ splenocytes were first stimulated with $20 \mathrm{ng} / \mathrm{ml}$ PMA and $2 \mu \mathrm{g} / \mathrm{ml}$ ionomycin for 3 hours in brefeldin A (BD Biosciences) at $37^{\circ} \mathrm{C}$ in complete RPMI- 1640 media. Cells were labeled for extracellular CD4 and CD25, treated with fixation/ permeabilization buffer (eBioscience), and then stained with antibodies for FOXP3 and IFN- $\gamma$ according to the manufacturer's specifications.

Renal analysis and histology. Urinalysis was performed with Uristix assays. A proteinuria measurement corresponding to $300 \mathrm{mg} / \mathrm{dl}$ or greater was considered a positive test. Leukocyte esterase content in the urine that corresponded with 10 or more leukocytes per microliter was considered a positive test. Renal function was assessed by the BUN content in serum, using a kinetic assay (Teco Diagnostics). For histology analysis, kidneys from 36- to 40-week-old mice were fixed in $10 \%$ neutral buffered formalin, and H\&E-stained sections were prepared by the Yale University Pathology Histology Service (New Haven, Connecticut, USA). Tissues were imaged on a Nikon TE-2000U microscope with a Nikon DS Fi1 color camera and NIS Elements AR software (version 2.30). Glomerular damage was scored on a scale of 0 to 6 , and intertubular infiltration from 0 to 3 , where a low score indicates less disease severity.

Antibody ELISAs. For dsDNA ELISAs, a coating layer of $10 \mu \mathrm{g} / \mathrm{ml}$ of methylated BSA (Calbiochem) in bicarbonate buffer ( $\mathrm{pH} 9.5$ ) was adsorbed onto high-binding 96-well plates, followed by incubation with $10 \mu \mathrm{g} / \mathrm{ml}$ of calf thymus DNA (Sigma-Aldrich). BSA (1\%) was used as a blocking agent and diluent, and $0.05 \%$ Tween-20 in PBS was used as a wash buffer. HRP-conjugated antibodies for mouse-specific pan-IgG (ICL Laboratories), IgG1, IgG2a, IgG2b, and IgG3 (Invitrogen) were used for detection, followed by TMB (KPL) substrate development.

Particle biodistribution studies. Nanogels were loaded with rhodamine and then injected intraperitoneally into mice. For organ biodistribution and histology studies, 12-week-old NZB/W F1 mice were used. Organs were harvested, weighed, and imaged with the IVIS imaging system (PerkinElmer) to quantify rhodamine fluorescence. For histological analysis, spleens were snap-frozen in OCT embedding medium (Sakura) and then sectioned at 5- $\mu \mathrm{m}$ thickness. Samples were fixed in ice-cold acetone for 10 minutes and subsequently stained with antibodies for CD4, CD19, or F4/80. Immunofluorescent histological samples were imaged on a Nikon TE-2000 microscope with a Roper Cool SNAP EZ camera using the following dichroic filters (bandwidths indicated): FITC channel (470/40,
525/50); rhodamine channel $(540 / 25,605 / 55)$; and Alexa647 channel $(620 / 60,700 / 75)$. For flow cytometric measurement of particle binding to immune cells, the analysis was performed in C57BL/6 mice immunized with $1 \times 10^{6}$ sheep red blood cells (Colorado Serum Company). Seven days after immunization, rhodamine-labeled particles were injected, and 2-3 hours later, cells from the spleen, lymph nodes (pooled inguinal, brachial, and cervical), and bone marrow were harvested and analyzed the same day on an LSR II flow cytometer.

Studies of nanogel interactions and effects on DCs. Bone marrow cells were isolated from the femurs and tibias of BALB/c mice. CD11 $\mathrm{c}^{+} \mathrm{DCs}$ were cultured from bone marrow cells using $10 \mathrm{ng} / \mathrm{ml}$ GM-CSF (eBioscience) and $5 \mathrm{ng} / \mathrm{ml}$ IL-4 (eBioscience) in RPMI-1640 complete media. On day 1 of the culture (24 hours after initial plating), $125 \mathrm{ng} / \mathrm{ml}$ MPA was added. Media was changed thereafter every 2 days with fresh MPA-loaded particles in the media. On day 6 of the culture, cells were replated at 200,000 cells per well in a 96-well round-bottom plate, and then challenged with $50 \mathrm{ng} /$ $\mathrm{ml}$ LPS (Sigma-Aldrich; catalog no. L2630) for 18 hours to induce maturation. The $\mathrm{CD} 11 \mathrm{c}^{+}$cell purity after 7 days was approximately $70 \%$. For mixed lymphocyte reactions, DCs were irradiated with $30 \mathrm{~Gy}$ in an X-Rad 320 (Precision XRay) and then cultured with CD4 T cells purified from $\mathrm{C} 57 \mathrm{BL} / 6$ mice. Cell purification was performed by magnetic separation (StemCell Technologies).

Samples for confocal imaging were prepared by seeding 7-day-old BMDCs onto ProbeOn Plus glass slides (Fisher Scientific), incubating with lissamine-rhodamine-labeled particles for 1 hour, and then staining cells with Alexa 488-phalloidin and TO-PRO-3 (Invitrogen). Cells were imaged with an LSM 510 Meta confocal microscope (Carl Zeiss)

For plasmacytoid DC studies, bone marrow cells were incubated with particles or free MPA for 30 minutes at $37^{\circ} \mathrm{C}$ in RPMI- 1640 complete media, washed, and then challenged with $1 \mu \mathrm{M}$ CpG-A oligonucleotide (ODN 1585; InvivoGen) for 18 hours.

Cytokine analysis. ELISAs for IL-12 p70 and TNF- $\alpha$ (eBioscience) and IFN- $\gamma$ (BD Biosciences) were performed according to the manufacturer's protocols. ELISAs for IFN- $\alpha$ were performed as described by Lund (46).

Toxicology studies. Acute toxicity studies were performed in 10-week-old C57BL/ 6 female mice. Mice were dosed with indicated treatment groups on days $0,1,2$, and 3 . Serum concentrations of ALKP, ALT, tBIL, and BUN were measured using reagents from Teco Diagnostics. Blood was collected in EDTA spray-coated tubes and immediately analyzed by $\mathrm{CBC}$ on a Hemavet blood counter (Drew Scientific). Urine was collected by bladder compression and analyzed with a Uristix test.

Statistics. All statistical analyses were performed using GraphPad Prism software (version 5.03). For comparisons in survival studies, the Mantel-Cox log-rank test was used. Experimental comparisons with multiple groups used ANOVA analysis with Bonferroni's post test. Two-tailed Student's $t$ tests were performed for some comparisons, as indicated in the figure captions. A $P$ value of 0.05 or less was considered statistically significant.

Study approval. All animal studies were performed with the approval of the IACUC of Yale University.

\section{Acknowledgments}

M. Look is a graduate research fellow of the National Defense Science and Engineering Graduate (NDSEG) program of the U.S. Department of Defense, and the National Science Foundation Graduate Research Fellowship Program (NSF GRFP). We thank Christoph Rahner and Morven Graham for assistance with transmission electron microscopy; Jonathan Alderman and Richard Flavell for use of the IVIS imaging system; Arunima Bandyopadhyay and Stacey Demento for technical advice on bone marrow DC cultures; Jason Criscione and Ragy Ragheb for technical advice 
on nanoparticle and polymer synthesis; Orlando Soto-Cáceres for assistance with in vitro studies; and Becky Robinson for additional advice with histology and photopolymerization. W. Mark Saltzman provided thoughtful critique. We also thank George Tsokos for review of this work and the Craft Lab for outstanding technical assistance and discussion. The authors would like to acknowledge the generous contributions of two foundations that made this work possible: the Wallace H. Coulter Foundation award (to T.M. Fahmy), and the Lupus Research Institute grant (to T.M. Fahmy and J. Craft). This work was also supported in part by NIH grants AR40072, AR44076, and AI075157 (to J. Craft).

Received for publication July 19, 2012, and accepted in revised form January 3, 2013.

Address correspondence to: Tarek M. Fahmy, 55 Prospect Street, MEC 412, New Haven, Connecticut 06520, USA. Phone: 203.432.1043; Fax: 203.432.0030; E-mail: tarek.fahmy@yale.edu.
1. Rahman A, Isenberg DA. Systemic lupus erythematosus. N Engl J Med. 2008;358(9):929-939.

2. Shlomchik MJ, Craft JE, Mamula MJ. From T to B and back again: positive feedback in systemic autoimmune disease. Nat Rev Immunol. 2001;1(2):147-153.

3. Teichmann LL, Ols ML, Kashgarian M, Reizis B, Kaplan DH, Shlomchik MJ. Dendritic cells in lupus are not required for activation of $\mathrm{T}$ and $\mathrm{B}$ cells but promote their expansion, resulting in tissue damage. Immunity. 2010;33(6):967-978.

4. Bennett L, et al. Interferon and granulopoiesis signatures in systemic lupus erythematosus blood. J Exp Med. 2003;197(6):711-723.

5. Jego G, Palucka AK, Blanck JP, Chalouni C, Pascual $\mathrm{V}$, Banchereau J. Plasmacytoid dendritic cells induce plasma cell differentiation through type I interferon and interleukin 6. Immunity. 2003;19(2):225-234.

6. Saxena R, Mahajan T, Mohan C. Lupus nephritis: current update. Arthritis Res Ther. 2011;13(5):240.

7. Contreras G, et al. Sequential therapies for proliferative lupus nephritis. $N$ Engl J Med. 2004; 350(10):971-980.

8. Petri M. Cyclophosphamide: new approaches for systemic lupus erythematosus. Lupus. 2004; 13(5):366-371.

9. Chatham WW, Kimberly RP. Treatment of lupus with corticosteroids. Lupus. 2001;10(3):140-147.

10. de Achaval S, Suarez-Almazor ME. Treatment adherence to disease-modifying antirheumatic drugs in patients with rheumatoid arthritis and systemic lupus erythematosus. Int J Clin Rheumtol. 2010;5(3):313-326.

11. Merrill JT, et al. The efficacy and safety of abatacept in patients with non-life-threatening manifestations of systemic lupus erythematosus: results of a twelve-month, multicenter, exploratory, phase IIb, randomized, double-blind, placebo-controlled trial. Arthritis Rheum. 2010;62(10):3077-3087.

12. Rovin BH, et al. Efficacy and safety of rituximab in patients with active proliferative lupus nephritis: the Lupus Nephritis Assessment with Rituximab study. Artbritis Rheum. 2012;64(4):1215-1226.

13. Navarra SV, et al. Efficacy and safety of belimumab in patients with active systemic lupus erythematosus: a randomised, placebo-controlled, phase 3 trial. Lancet. 2011;377(9767):721-731.

14. Blanco E, Hsiao A, Mann AP, Landry MG, Meric-Bernstam F, Ferrari M. Nanomedicine in cancer therapy: innovative trends and prospects. Cancer Sci. 2011;102(7):1247-1252.

15. Look M, Bandyopadhyay A, Blum JS, Fahmy TM. Application of nanotechnologies for improved immune response against infectious diseases in the developing world. Adv Drug Deliv Rev. 2010; 62(4-5):378-393.

16. Scindia Y, Deshmukh U, Thimmalapura PR, Bagavant $H$. Anti-alpha8 integrin immunoliposomes in glomeruli of lupus-susceptible mice: a novel system for delivery of therapeutic agents to the renal glomerulus in systemic lupus erythematosus. Arthritis Rheum. 2008;58(12):3884-3891.

17. Serkova NJ, et al. Renal inflammation: targeted iron oxide nanoparticles for molecular MR imaging in mice. Radiology. 2010;255(2):517-526.

18 . Stern E, et al. Combination delivery of TGF- $\beta$ inhibitor and IL-2 by nanoscale liposomal polymeric gels enhances tumor immunotherapy. Nat Mater. 2012;11(10):895-905.

19. Kabanov AV, Vinogradov SV. Nanogels as pharmaceutical carriers: finite networks of infinite capabilities. Angew Chem Int Ed Engl. 2009;48(30):5418-5429.

20. Maurer N, Fenske DB, Cullis PR. Developments in liposomal drug delivery systems. Expert Opin Biol Ther. 2001;1(6):923-947.

21. Hillery AM, Lloyd AW, Swarbrick J. Drug Delivery and Targeting for Pharmacists and Pharmacentical Scientists. New York, New York, USA: Taylor and Francis; 2001.

22. Appel GB, et al. Mycophenolate mofetil versus cyclophosphamide for induction treatment of lupus nephritis. J Am Soc Nephrol. 2009;20(5):1103-1112.

23. Dooley MA, et al. Mycophenolate versus azathioprine as maintenance therapy for lupus nephritis. NEngl J Med. 2011;365(20):1886-1895.

24. Elbarbry FA, Shoker AS. Liquid chromatographic determination of mycophenolic acid and its metabolites in human kidney transplant plasma: pharmacokinetic application.J Chromatogr B Analyt Technol Biomed Life Sci. 2007;859(2):276-281.

25. Sawhney AS, Pathak CP, Hubbell JA. Bioerodible hydrogels based on photopolymerized poly(ethylene glycol)-co-poly( $\alpha$-hydroxy acid) diacrylate macromers. Macromolecules. 1993;26:581-587.

26. Linterman MA, et al. Follicular helper T cells are required for systemic autoimmunity. J Exp Med. 2009;206(3):561-576.

27. Odegard JM, et al. ICOS-dependent extrafollicular helper T cells elicit IgG production via IL-21 in systemic autoimmunity. J Exp Med. 2008; 205(12):2873-2886

28. Masutani K, et al. Predominance of Th1 immune response in diffuse proliferative lupus nephritis. Arthritis Rheum. 2001;44(9):2097-2106.

29. Wofsy D, Seaman WE. Successful treatment of autoimmunity in NZB/NZW F1 mice with monoclonal antibody to L3T4. J Exp Med. 1985;161(2):378-391.

30. Wofsy D, Seaman WE. Reversal of advanced murine lupus in NZB/NZW F1 mice by treatment with monoclonal antibody to L3T4. J Immunol. 1987; 138(10):3247-3253.

31. Bekar KW, et al. Prolonged effects of short-term anti-CD20 B cell depletion therapy in murine systemic lupus erythematosus. Arthritis Rheum. 2010; 62(8):2443-2457.

32. Li Y, et al. B cell depletion with anti-CD79 mAbs ameliorates autoimmune disease in MRL/lpr mice.
JImmunol. 2008;181(5):2961-2972.

33. Ahuja A, Shupe J, Dunn R, Kashgarian M, Kehry MR, Shlomchik hMJ. Depletion of B cells in murine lupus: efficacy and resistance. J Immunol. 2007; 179(5):3351-3361.

34. Ramos MA, et al. Modulation of autoantibody production by mycophenolate mofetil: effects on the development of SLE in (NZB x NZW)F1 mice. Nephrol Dial Transplant. 2003;18(5):878-883.

35. Lui SL, et al. Effect of mycophenolate mofetil on severity of nephritis and nitric oxide production in lupus-prone MRL/lpr mice. Lupus. 2002; 11(7):411-418.

36. Mehling A, Grabbe S, Voskort M, Schwarz T, Luger TA, Beissert S. Mycophenolate mofetil impairs the maturation and function of murine dendritic cells. J Immunol. 2000;165(5):2374-2381.

37. Lagaraine C, Hoarau C, Chabot V, Velge-Roussel F, Lebranchu Y. Mycophenolic acid-treated human dendritic cells have a mature migratory phenotype and inhibit allogeneic responses via direct and indirect pathways. Int Immunol. 2005;17(4):351-363.

38. Shirali AC, et al. Nanoparticle delivery of mycophenolic acid upregulates PD-L1 on dendritic cells to prolong murine allograft survival. Am J Transplant. 2011;11(12):2582-2592.

39. Ginzler EM, et al. Mycophenolate mofetil or intravenous cyclophosphamide for lupus nephritis. NEngl J Med. 2005;353(21):2219-2228.

40. Jacob CO, et al. Pivotal role of Stat 4 and Stat 6 in the pathogenesis of the lupus-like disease in the New Zealand mixed 2328 mice. J Immunol. 2003; 171(3):1564-1571.

41. Steinmetz OM, et al. CXCR3 mediates renal Th1 and Th17 immune response in murine lupus nephritis. J Immunol. 2009;183(7):4693-4704.

42. Peng SL, Moslehi J, Craft J. Roles of interferon-gamma and interleukin-4 in murine lupus. J Clin Invest. 1997;99(8):1936-1946.

43. Ichinose K, et al. Cutting edge: calcium/calmodulin-dependent protein kinase type IV is essential for mesangial cell proliferation and lupus nephritis. J Immunol. 2011;187(11):5500-5504.

44. Ziswiler R, Steinmann-Niggli K, Kappeler A, Daniel C, Marti HP. Mycophenolic acid: a new approach to the therapy of experimental mesangial proliferative glomerulonephritis. J Am Soc Nephrol. 1998; 9(11):2055-2066.

45. Suana AJ, et al. Single application of low-dose mycophenolate mofetil-OX7-immunoliposomes ameliorates experimental mesangial proliferative glomerulonephritis. J Pharmacol Exp Ther. 2011; 337(2):411-422.

46. Lund J, Sato A, Akira S, Medzhitov R, Iwasaki A. Toll-like receptor 9-mediated recognition of herpes simplex virus- 2 by plasmacytoid dendritic cells. JExp Med. 2003;198(3):513-520. 